\title{
DSMC simulation of gas heat transfer between coaxial cylinders
}

Cite as: AIP Conference Proceedings 2132, 070011 (2019); https://doi.org/10.1063/1.5119565

Published Online: 05 August 2019

Hang Song, Chao Yang, and Quanhua Sun

ARTICLES YOU MAY BE INTERESTED IN

A microscopic study of one-dimensional $\mathrm{H}_{2}-\mathrm{O}_{2}$ detonation using DSMC method

AIP Conference Proceedings 2132, 070013 (2019); https://doi.org/10.1063/1.5119567

DSMC simulations of turbulent flows at moderate Reynolds numbers

AIP Conference Proceedings 2132, 070010 (2019); https://doi.org/10.1063/1.5119564

Validation of DSMC and NS computations for high-enthalpy non-equilibrium flows in ground and flight tests

AIP Conference Proceedings 2132, 070007 (2019); https://doi.org/10.1063/1.5119561

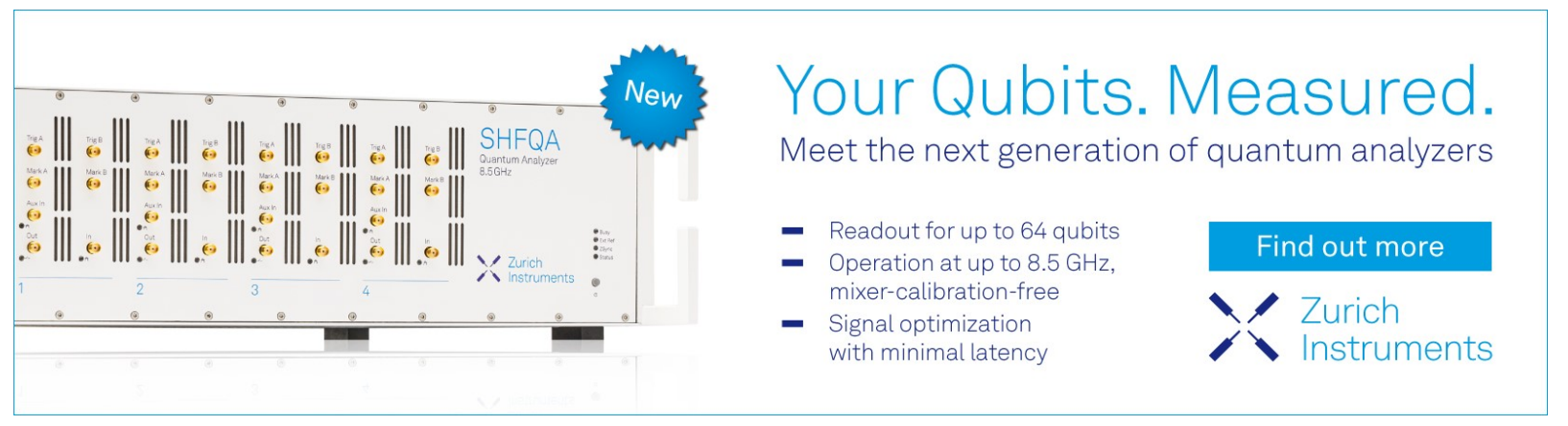




\title{
DSMC Simulation of Gas Heat Transfer Between Coaxial Cylinders
}

\author{
Hang Song ${ }^{1,2, a)}$, Chao Yang, b) and Quanhua Sun ${ }^{1,2, c)}$ \\ ${ }^{1}$ State Key Laboratory of High Temperature Gas Dynamics, Institute of Mechanics, CAS, Beijing 100190, China \\ ${ }^{2}$ School of Engineering Science, University of Chinese Academy of Sciences, Beijing 100049, China \\ a)Songhang@imech.ac.cn \\ b)yangchao@imech.ac.cn \\ ${ }^{c)}$ qsun@imech.ac.cn
}

\begin{abstract}
How to reduce the heat loss caused by heat conduction of gas between coaxial cylinders is of vital importance. The problem is widely presented in the oil transportation pipeline and the solar parabolic trough receiver, where hydrogen percolation is inevitable. One of the most common methods is to fill with inert gas, such as argon and xenon. In this work, several factors affecting the heat loss in the coaxial cylinders are studied using the DSMC method, including the wall thermal accommodation coefficient, wall temperature, gas composition, and number density of the gas mixture. After determining argon as the filling gas, the effect of different combinations of the argon and hydrogen densities on the heat flux was studied, and an empirical formula was proposed to evaluate the heat flux of the $\mathrm{Ar}-\mathrm{H}_{2}$ mixture.
\end{abstract}

\section{INTRODUCTION}

The heat transfer of gas between coaxial cylinders at various pressures is a classical problem in gas dynamics. It exists in many industrial applications, such as oil transportation where argon gas is filled between insulated pipelines to prevent heat loss, and solar energy collection where xenon gas is filled in the solar parabolic trough receiver. This problem has been widely studied in the literature, especially for solar parabolic trough receiver. For instance, O'shea and Collins [1] measured the heat flux rate of parabolic trough receivers filled with different kinds of pure gases and mixtures under different Knudsen numbers. Empirical formulae were employed or presented to predict the heat flux such as Sherman formula [3]. In addition, DSMC simulations were able to show good results as compared with the experimental data [1]. In this study, the general behavior of heat transfer between coaxial cylinders is investigated using the DSMC method. In order to reduce the heat loss in oil pipelines, the mixture of argon and hydrogen is especially analyzed where hydrogen with a high thermal conductivity penetrates into the thermal insulation layer of pipelines.

\section{PROBLEM DESCRIPTION AND NUMERICAL SETUP}

The physical problem is described in Fig. 1 . The radius of inner cylinder $\left(R_{1}\right)$ is $0.1 \mathrm{~m}$ and the radius of outer cylinder $\left(R_{2}\right)$ is $0.2 \mathrm{~m}$. The other boundary and initial conditions are as follows:

(1) The outer cylinder is fixed at $T_{2}=273 \mathrm{~K}$, and the default value of inner cylinder is $T_{1}=323 \mathrm{~K}$.

(2) The thermal accommodation coefficients of cylinder surface $\left(\alpha_{1}\right.$ and $\left.\alpha_{2}\right)$ are 1.0 unless otherwise specified.

(3) The gas between the cylinders is specified using the averaged number density.

As the problem is actually axisymmetric along the coaxial, flows at small Knudsen numbers are simulated using a sector where periodic boundary condition is adopted to save computational cost and to avoid using particle weight scheme in the DSMC method. The setup for a sector is shown in Fig. 1(b).

31st International Symposium on Rarefied Gas Dynamics

AIP Conf. Proc. 2132, 070011-1-070011-8; https://doi.org/10.1063/1.5119565

Published by AIP Publishing. 978-0-7354-1874-5/\$30.00 


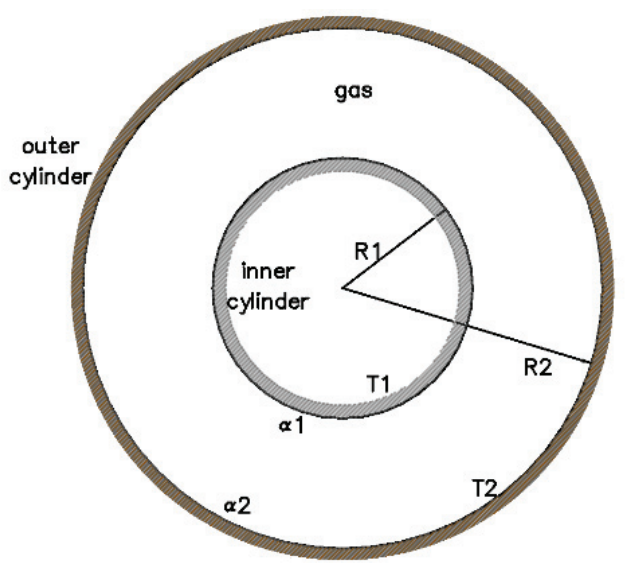

(a)

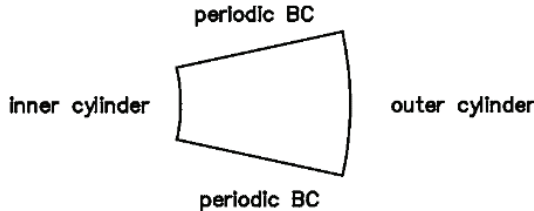

(b)

Figure 1. Schematic of gas heat transfer between coaxial cylinders. (a) full cylinders, (b) setup for small $K n$ cases $(K n<0.1)$.

\section{RESULTS AND DISCUSSION}

The DSMC method employs the VHS model for molecular collisions and the Larsen-Borgnakke model for energy transfer in inelastic collisions. Simulations using the VSS model are also tested but show no obvious difference from the results using the VHS model under the current conditions where the temperature variation for all cases is no more than $60 \mathrm{~K}$. It is unclear why the molecular model plays little effect on the heat flux, which requires further investigation.

The bridging formula of Sherman has been successfully applied to many rarefied gas flows and is also tested for the current problem. According to the Sherman formula, the heat transfer between the coaxial cylinders is shown as follows,

$$
\begin{gathered}
\frac{1}{q_{\text {transition }}}=\frac{1}{q_{\text {FreeMolecular }}}+\frac{1}{q_{\text {Continuum }}}, \\
q_{\text {FreeMolecular }}=\frac{1}{\frac{1}{\alpha_{1}}+\left(\frac{R_{1}}{R_{2}}\right)\left(\frac{1}{\alpha_{2}}-1\right)} \frac{n k_{B} \bar{T}\left(\frac{R_{g}}{\gamma-1}+\frac{R_{g}}{2}\right)\left(T_{1}-T_{2}\right)}{\sqrt{2 \pi R_{g} \bar{T}}}, \\
q_{\text {continum }}=\frac{k_{\text {gas }}\left(T_{1}-T_{2}\right)}{R_{1} \ln \left(\frac{R_{2}}{R_{1}}\right)},
\end{gathered}
$$

where $\bar{T}=\left(T_{1}+T_{2}\right) / 2$.

The heat flux on the inner cylinder is plotted in Fig. 2 from near continuum $\left(K n=0.01, n=1.23 \times 10^{21} \mathrm{~m}^{-3}\right)$ to free molecular $\left(K n=100, n=1.23 \times 10^{17} \mathrm{~m}^{-3}\right)$ flow for argon where the Sherman formula is also plotted. It shows that the heat flux increases with the gas number density and approaches to the theoretical value in the continuum limit $\left(12.48 \mathrm{~W} \cdot \mathrm{m}^{-2}\right)$. The agreement between DSMC result and Sherman formula is very good.

The heat flux for different gases is compared in Fig. 3, which shows that hydrogen and helium have strong thermal conductivity whereas xenon has strong thermal insulation capability. Argon is less effective for insulation than xenon but it is relatively cheap economically.

Theoretically, the heat flux at free molecular is proportional to the square root of the gas constant as shown in Eq. (2), but the behavior of heat flux at the continuum limit is more complicated. Actually, Eq. (3) can be rewritten as Eq. (4) by relating the heat conduct coefficient with the viscosity, which shows that the heat flux at the continuum limit depends on the gas constant, number of degrees of freedom (or ratio of specific heats), and viscosity of filled gas. 


$$
q_{\text {continuum }}=\frac{k_{\text {gas }}\left(T_{1}-T_{2}\right)}{R_{1} \ln \left(\frac{R_{2}}{R_{1}}\right)}=\frac{\gamma}{\gamma-1} \frac{R_{u}}{M} \frac{\mu}{\operatorname{Pr}} \frac{\left(T_{1}-T_{2}\right)}{R_{1} \ln \left(\frac{R_{2}}{R_{1}}\right)} .
$$

It can be concluded now from equation (1) that the main reasons affecting the heat flux of different gases are the ratio of specific heats $\gamma$, relative atomic mass $M$ and viscosity coefficient $\mu$. The heat flux increases as the relative atomic mass and the specific heat ratio decrease, and increases with the viscosity coefficient increases. Currently, monatomic gases have lower heat conductivity than diatomic and polyatomic gases, the heat conductivity of gases with lower relative atomic mass are higher than the gases with higher relative atomic mass.

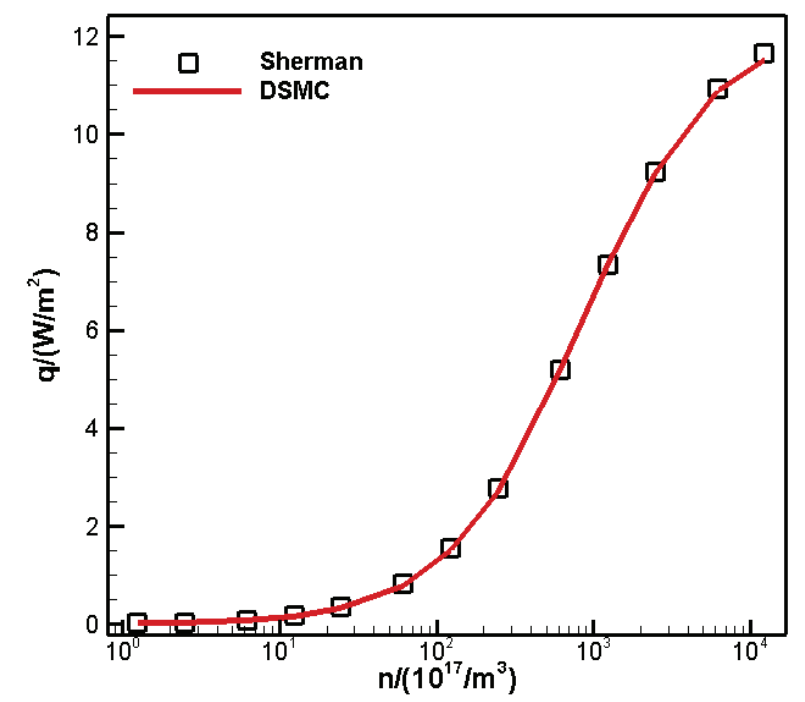

Figure 2. The result of DSMC simulation of argon comparing with Sherman formula

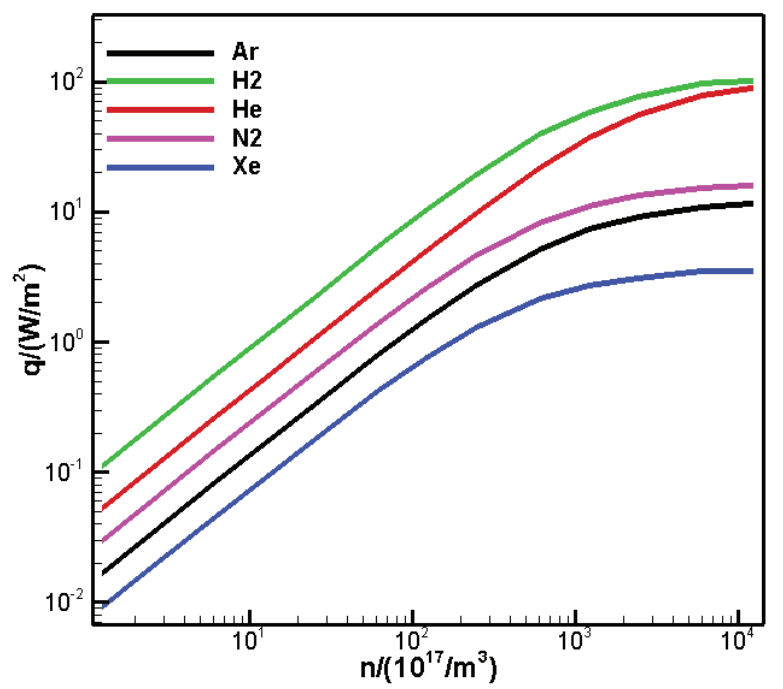

Figure 3. The heat flux for different gases

The effect of the temperature difference on the heat flux is shown in Fig. 4 for argon where Fig. 4(a) are the heat flux values and Fig. 4(b) shows the products of heat flux and the temperature difference. Clearly, the heat flux depends on the temperature difference and is proportional to the temperature difference at the present temperature range. 


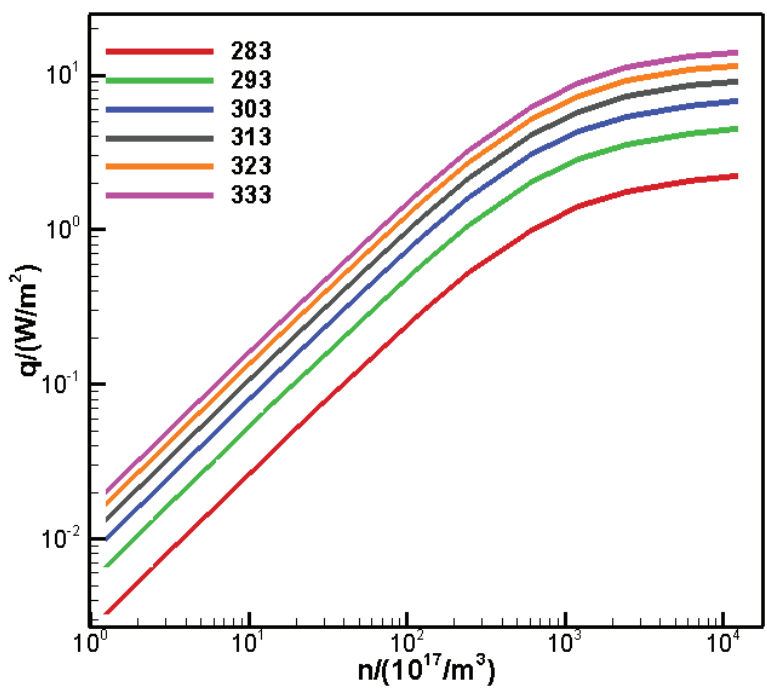

(a)

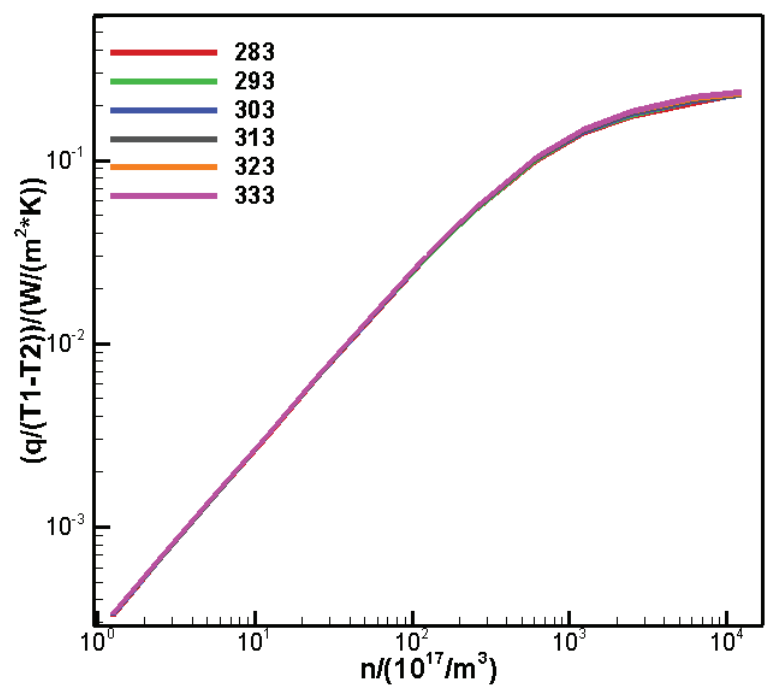

(b)

Figure 4. Heat flux at different inner wall temperatures

The effect of the thermal accommodation coefficient (TAC) is shown in Fig. 5, where the TAC of argon is between 0.8 and 1.0 whereas the TAC of hydrogen is between 0.2 and 1.0. Clearly, a small TAC can decrease the heat flux on the cylinder surface. Since the variation of the TAC of hydrogen is very large in engineering applications, it is desired to determine the value of the TAC of hydrogen in advance.

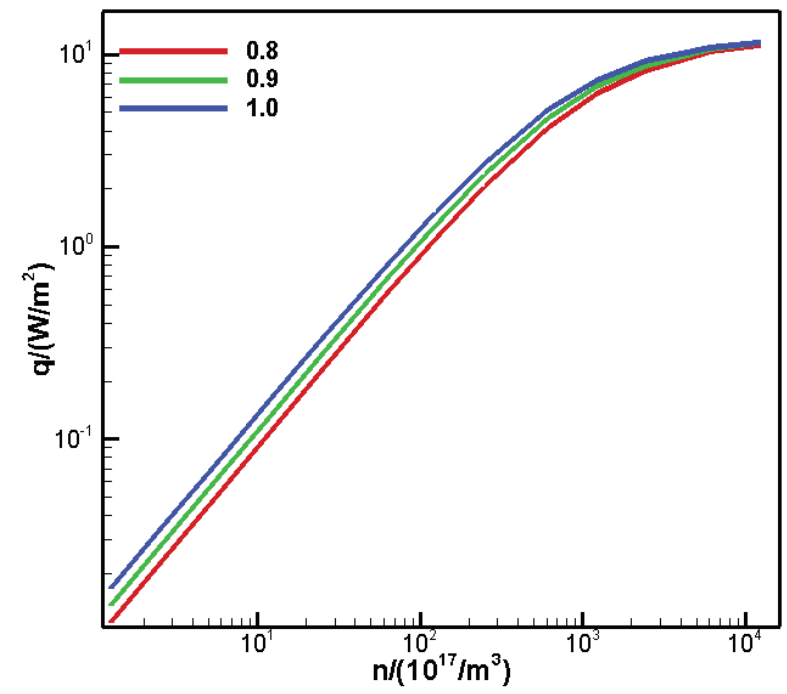

(a)

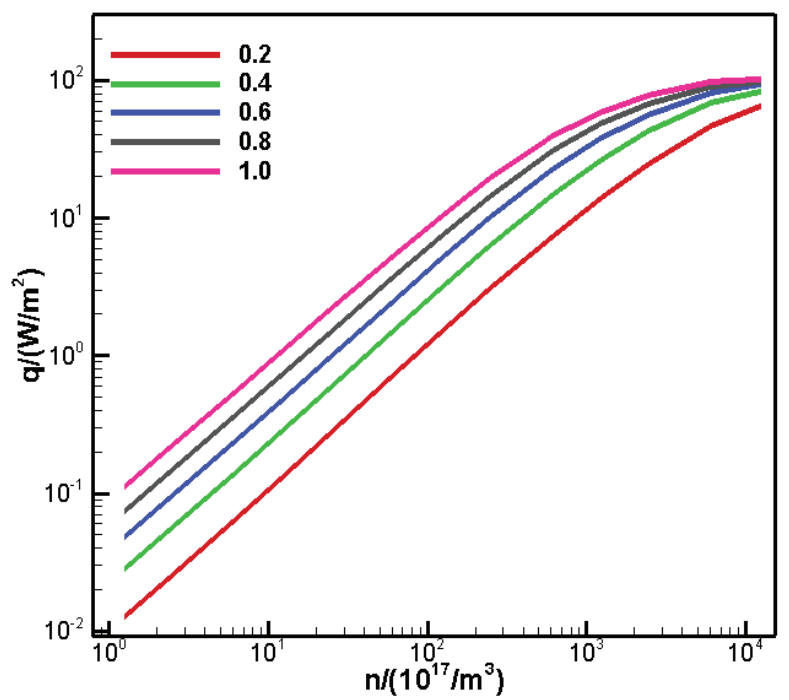

(b)

Figure 5. Influence of the wall thermal accommodation coefficient on surface heat flux. (a) TAC of Ar; (b) TAC of $\mathrm{H}_{2}$.

When to fill with noble gas between insulated pipelines to prevent heat loss? In the case when TACs of argon and hydrogen are 1.0, simulations have been performed for mixtures at different number densities. By varying the number density of hydrogen when the argon density is fixed, we can draw a conclusion from Fig. 6 that if the molecular density of hydrogen is lower than $1.23 \times 10^{19} \mathrm{~m}^{-3}$, a vacuum adiabatic pipe should be used to maintain a high vacuum of the insulation layer. In order to reach this goal, several means can be adopted such as increasing the degasser, using metals that are harder to dissolve the hydrogen, or reducing the concentration of hydrogen in the tube. 


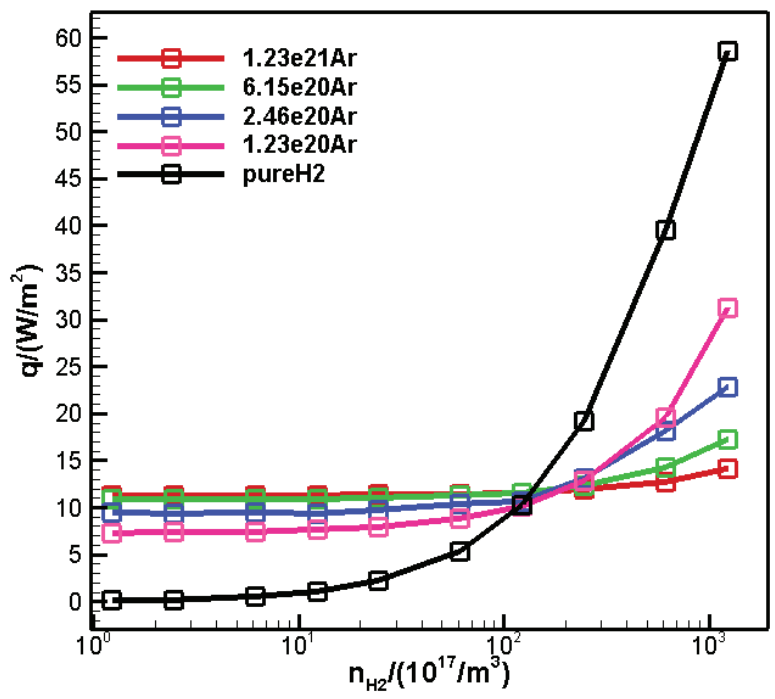

Figure 6. The heat transfer capacity of mixture at fixed argon molecular density

How much argon should be filled when the hydrogen density is fixed? Figure 7 shows that more argon is better for less heat loss when the hydrogen density is higher than $1.23 \times 10^{19} \mathrm{~m}^{-3}$. The reason for this phenomenon is that the molecular weight of hydrogen molecules is very small, the rate of molecular thermal motion is very high, and it is easy to collide with the wall surface, transferring the heat from the inner wall with higher temperature to the outer wall with lower temperature. The relative molecular mass of argon is relatively large, its molecular thermal motion rate is low, and heat transfer is relatively small. If hydrogen molecules collide with argon molecules, their trajectories will be deflected, increasing the time interval between the collision of hydrogen molecules and the two walls, thus reducing the heat flux.

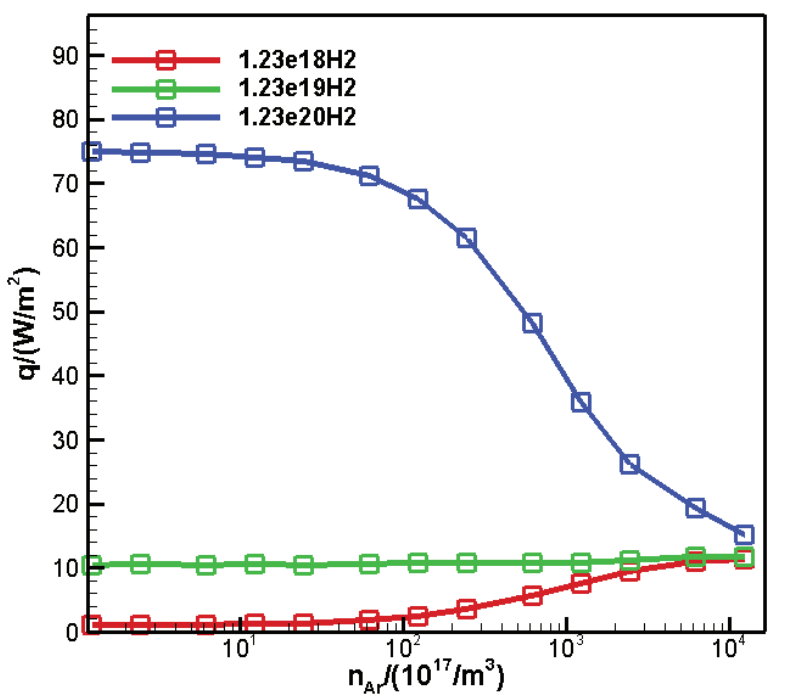

Figure 7. The heat transfer capacity of mixture at fixed hydrogen molecular density.

There are some methods to estimate the heat transfer of binary mixed gases such as the Wassiljewa equation [6]. In this paper, the Wassiljewa equation is used to calculate the heat flux of the mixed gas in the continuous domain, and the heat flux in the temperature jump and transition domains is calculated by interpolation of Sherman equation. The Wassiljewa equation evaluates the thermal conductivity of the mixture as follows, 


$$
k=\sum_{i=1}^{n} \frac{x_{i} k_{i}}{\sum_{j=1}^{n} x_{j} A_{i j}},
$$

where $k$ is the thermal conductivity of the mixture, $x_{\mathrm{i}}$ and $k_{i}$ are the molecular fraction and the thermal conductivity for each component, $A_{\mathrm{ij}}$ are the binding parameters which can be calculated as below.

$$
A_{i j}=\left[1+\left(\frac{\mu_{i}}{\mu_{j}}\right)^{0.5}\left(\frac{M_{j}}{M_{i}}\right)^{0.25}\right]^{2}\left(\sqrt{8}\left(1+\frac{M_{i}}{M_{j}}\right)^{0.5}\right)^{-1} .
$$

The heat flux of gases in the continuous domain can be calculated using the formula (3). Since the hydrogen and argon molecules in the free molecular state never collide with other molecules, the heat flux in the free molecular flow can be calculated through the following equation:

$$
\begin{aligned}
& q_{\text {Freemolecular }}=q_{A r}+q_{H 2} \\
& =\frac{n_{A r} k_{B} \bar{T}\left(\frac{R_{A r}}{\gamma_{A r}-1}+\frac{R_{A r}}{2}\right)\left(T_{1}-T_{2}\right)}{\sqrt{2 \pi R_{A r} \bar{T}}}+\frac{n_{H 2} k_{B} \bar{T}\left(\frac{R_{H 2}}{\gamma_{H 2}-1}+\frac{R_{H 2}}{2}\right)\left(T_{1}-T_{2}\right)}{\sqrt{2 \pi R_{H 2} \bar{T}}} .
\end{aligned}
$$

Figure 8 shows the comparison of heat fluxes from the Sherman formula (3) using the Wassiljewa equation and the DSMC simulation results of the $\mathrm{Ar}-\mathrm{H}_{2}$ mixture at different numbers. It shows that the formula works well when the number density of argon is much larger than that of hydrogen. However, when the number density of hydrogen is close to that of argon, the difference between the DSMC results and the formula data increases gradually.

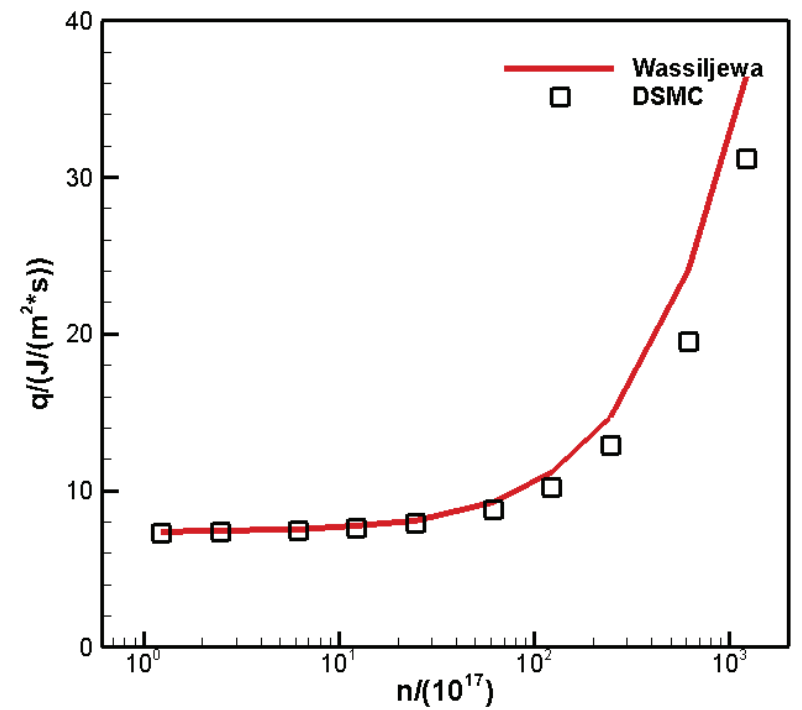

(a)

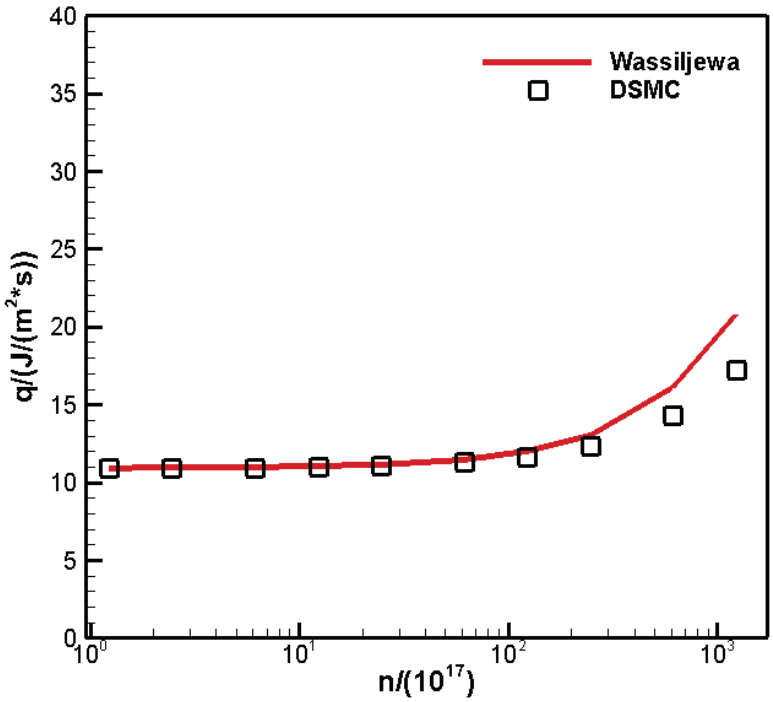

(b)

Figure 8. Comparisons of heat flux using the Wassiljewa equation and the simulation results using the DSMC method at different hydrogen number density. (a) the molecular number of prefilled argon is $1.23 \times 10^{20} \mathrm{~m}^{-3}$, (b) the molecular number of prefilled argon is $6.15 \times 10^{20} \mathrm{~m}^{-3}$.

In order to obtain a better fitting formula, the Sherman formula is modified. First, the value of heat flux in pure $\operatorname{argon}$ with $n_{A r}$ in the mixed gas is set as $q_{A r}$, which is evaluated according to the Sherman formula,

$$
\begin{gathered}
\frac{1}{q_{A r}}=\frac{1}{q_{A r F M}}+\frac{1}{q_{\text {Arcontinum }}}, \\
q_{A r F M}=\frac{n_{A r} k_{B} \bar{T}\left(\frac{R_{A r}}{\gamma_{A r}-1}+\frac{R_{A r}}{2}\right)\left(T_{1}-T_{2}\right)}{\sqrt{2 \pi R_{A r} \bar{T}}},
\end{gathered}
$$




$$
q_{\text {Arcontinuum }}=\frac{k_{A r}\left(T_{1}-T_{2}\right)}{R_{1} \ln \left(\frac{R_{2}}{R_{1}}\right)} .
$$

Due to the presence of hydrogen gas, the heat flux of the mixed gas is larger than the corresponding pure argon value. Now taking the extra heat flux $q-q_{A r}$ into the Sherman formula and allows a free parameter $\beta$ in the free molecular term, we get the following formula,

$$
\begin{gathered}
\frac{1}{q-q_{A r}}=\beta \frac{1}{q_{H 2 F M}}+\frac{1}{q_{H 2 \text { continuum }}-q_{A r}}, \\
q_{H 2 F M}=\frac{n_{H 2} k_{B} \bar{T}\left(\frac{R_{H 2}}{\gamma_{H 2}-1}+\frac{R_{H 2}}{2}\right)\left(T_{1}-T_{2}\right)}{\sqrt{2 \pi R_{H 2} \bar{T}}} .
\end{gathered}
$$

Based on the DSMC data, the expression of $\beta$ can be formulated. When $n_{A r} \rightarrow 0, \beta \rightarrow 1$ and when $n_{A r} \rightarrow \infty$, $\beta \rightarrow \infty$. We then assume $\beta$ has a linear expression of the density as follows,

$$
\beta=2.1789 \times 10^{-20} \cdot n_{A r}+1 .
$$

The new formula for predicting the heat flux of $\mathrm{Ar}-\mathrm{H}_{2}$ mixture is shown in Fig. 7, which shows the agreement between DSMC and the formula is very good.
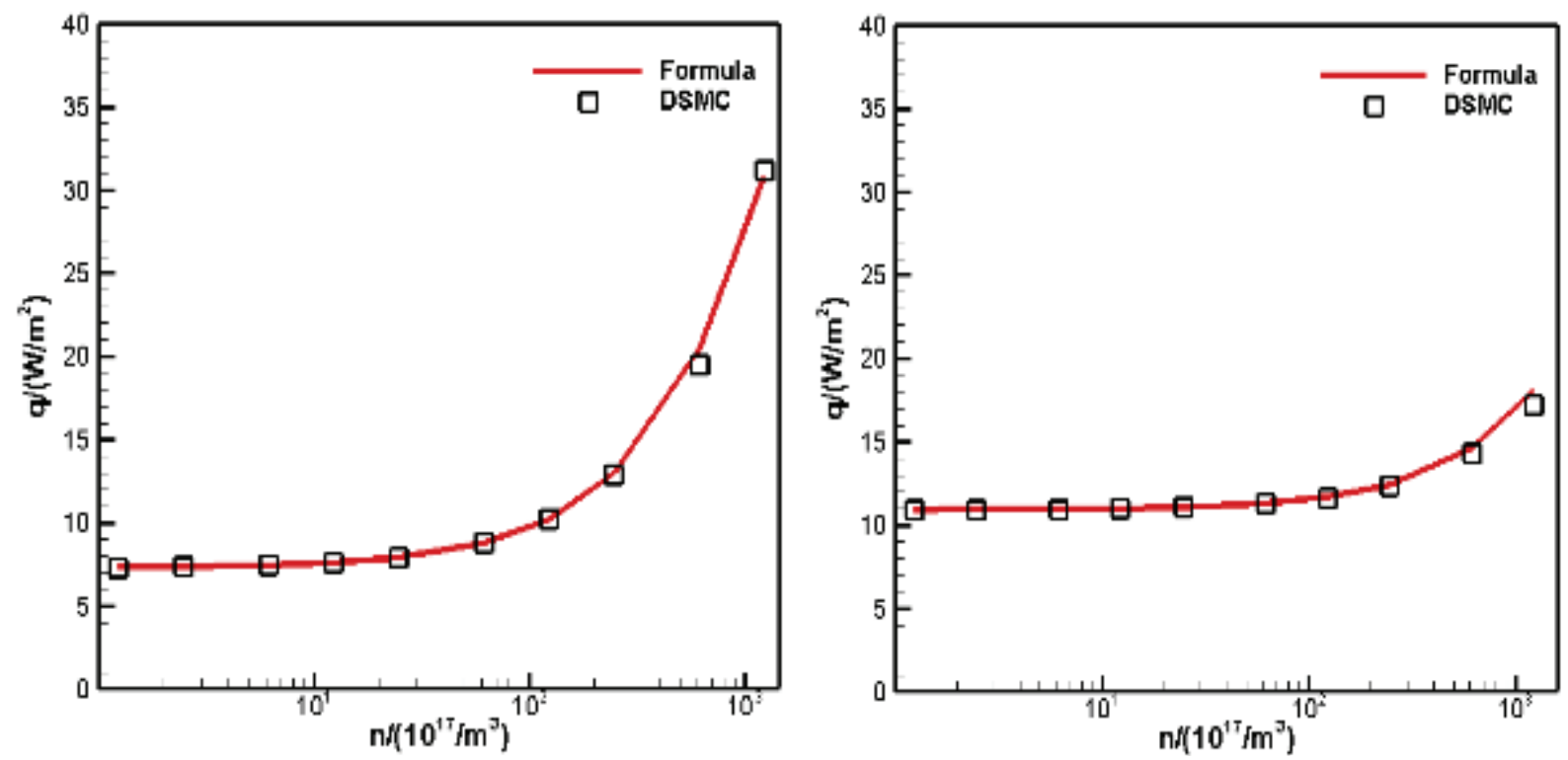

Figure 9. The calculation results of new formula are compared with that of DSMC simulation. (a) The molecular number density of prefilled argon is $1.23 \times 10^{20} \mathrm{~m}^{-3}$; (b) The molecular number of prefilled argon is $6.15 \times 10^{20} \mathrm{~m}^{-3}$.

\section{CONCLUSION}

In this paper, the heat transfer between coaxial cylinders is investigated using the DSMC method. It is found that the bridging formula of Sherman predicts good heat flux for pure gases as compared with the DSMC simulation results. With the Sherman formula, it shows that the light gas conducts heat much better than the heavy gas, thus xenon is preferred as filling gas between pipes to prevent heat loss and argon may be the practical choice with low economic cost. The Sherman formula, however, failed to predict the heat flux of mixture at high number density. A new formula is then proposed by evaluating the heat flux modification to the pure argon value of the Sherman formula. It should be mentioned that the current DSMC simulations are mainly based on the VHS molecular model. Some VSS simulation results are very close to those of VHS data, which requires further investigation. 


\section{ACKNOWLEDGMENTS}

This work was supported by the Strategic Priority Research Program of Chinese Academy of Sciences (Grant No.XDA17030100) and the National Natural Science Foundation of China (Grants 11372325 and 91116013).

\section{REFERENCES}

1. S. J. O'shea and R. E. Collins. International journal of heat and mass transfer 35(12), 3431-3440 (1992).

2. M. Knudsen. The Kinetic Theory of Gases (London, 1934).

3. F. S. Sherman. "A survey of experimental results and methods for the transition regime of rarefied gas dynamics," in Rarefied Gas Dynamics, Proc. of the Third International Symposium on Rarefied Gas Dynamics, Paris, France, 1963, pp. 228-260.

4. G.A. Bird. Molecular Gas Dynamics and the Direct Simulation of Gas Flows (Clarendon, Oxford, 1994).

5. E. S. Udoetok. Frontiers in Heat and Mass Transfer 4(2), 023008 (2013).

6. M. P. Saksena and S. C. Saxena. Applied Scientific Research 17(4-5), 326-330 (1967).

7. B. J. McCoy and C. Y. Cha. Chemical Engineering Science 29(2), 381-388 (1974). 